\title{
Densely Packed Linear Assembles of Carbon Nanotube Bundles in Polysiloxane-Based Nanocomposite Films
}

\author{
Hong-Baek Cho, ${ }^{1}$ Minh Triet Tan Huynh, ${ }^{1}$ Tadachika Nakayama, ${ }^{1}$ Son Thanh Nguyen, ${ }^{1}$ \\ Hisayuki Suematsu, ${ }^{1}$ Tsuneo Suzuki, ${ }^{1}$ Weihua Jiang, ${ }^{1}$ Satoshi Tanaka, ${ }^{1}$ Yoshinori Tokoi, ${ }^{2}$ \\ Soo Wohn Lee, ${ }^{3}$ Tohoru Sekino, ${ }^{4}$ and Koichi Niihara ${ }^{1}$ \\ ${ }^{1}$ Extreme Energy-Density Research Institute, Nagaoka University of Technology, Nagaoka, Niigata 940-2188, Japan \\ ${ }^{2}$ Department of Electrical and Engineering System Engineering, Nagaoka National College of Technology, Nagaoka, \\ Niigata 940-8532, Japan \\ ${ }^{3}$ Department of Environment Engineering, Sun Moon University, Asan, Chung Nam 336-708, Republic of Korea \\ ${ }^{4}$ Institute of Multidisciplinary Research for Advanced Materials, Tohoku University, Sendai, Miyagi 980-8577, Japan
}

Correspondence should be addressed to Hong-Baek Cho; hong-baekcho@etigo.nagaokaut.ac.jp

Received 13 December 2012; Accepted 18 January 2013

Academic Editor: Jian Wei

Copyright (c) 2013 Hong-Baek Cho et al. This is an open access article distributed under the Creative Commons Attribution License, which permits unrestricted use, distribution, and reproduction in any medium, provided the original work is properly cited.

\begin{abstract}
Linear assemblies of carbon nanotubes (LACNTs) were fabricated and controlled in polysiloxane-based nanocomposite films and the effects of the LACNTs on the thermal and electrical properties of the films were investigated. CNTs were dispersed by mechanical stirring and sonication in a prepolymer of polysiloxane. Homogeneous suspensions were cast on polyamide spacers and oriented by linear-assembly by applying DC and switching DC electric fields before the mixture became cross-linked. Densely packed LACNTs that fixed the composite film surfaces were fabricated with various structures and thicknesses that depended on the DC and switching DC conditions. Polymer nanocomposites with different LACNT densities exhibited enhanced thermal and electrical conductivities and high optical transmittances. They are considered promising structural materials for electronic sectors in automotive and aerospace applications.
\end{abstract}

\section{Introduction}

Polymer-based nanocomposites, which are formed by adding a small amount of nanoparticles to a polymer matrix, have generated great interest in science and engineering because they exhibit superior mechanical, thermal, and electrical properties through controlled assembly of inclusions [1-4]. Polymers are multifunctional materials with superior properties, including reasonable strength, good processability, and high elasticity. Consequently, polymer-based materials are widely used in various applications including electrical devices, filtration, and biomedical applications [5]. However, polymers have very low thermal conductivities. It is thus necessary to increase the thermal conductivities of polymerbased materials for scientific and industrial applications. The physical properties of polymer-based materials have been improved by adding fillers with high thermal or electrical conductivities such as boron nitride (BN) $[6,7]$, carbon nanotubes (CNTs) [8-10], and graphite nanosheets [11] to polymer matrices. The thermal conductivities of such composites are strongly affected by the dispersion, orientation, and linear alignment of inorganic fillers aligned to parallel to the thermal flux [12-14] in polymer matrices [15]. The orientation and formation of network structures containing small quantities of nanofillers in a polymer matrix can be controlled by applying a shear force [16], a magnetic field [17], or an electric field [18-20], or by mechanical stretching $[21,22]$. Of these methods, orienting conducting or dielectric nanofillers by applying an electric field has the advantage that the fillers form self-assembled linear structures due to Coulombic attraction during electrophoretic movement in a polymer with a controlled viscosity $[23,24]$. Assembly of fillers with end-to-end attachment can also reduce the thermal boundary resistance. This cannot be achieved in 


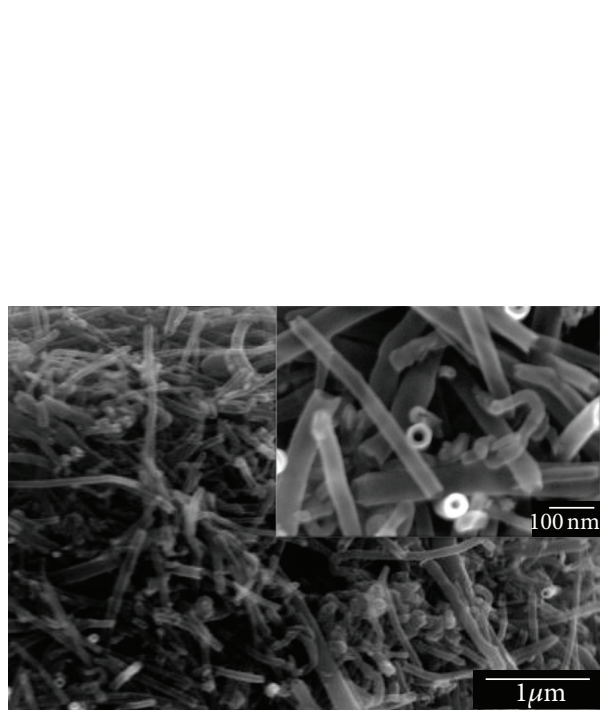

(a)

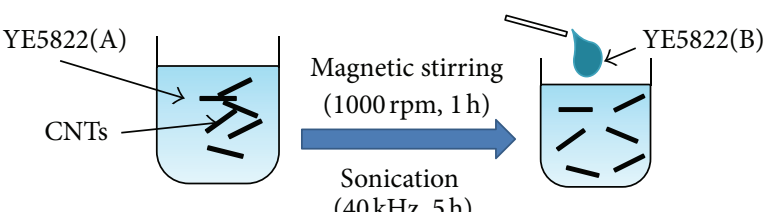

$(40 \mathrm{kHz}, 5 \mathrm{~h})$
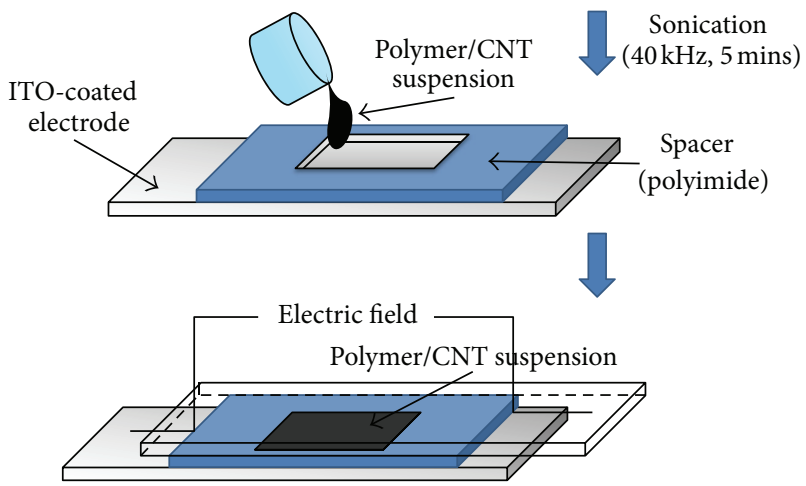

(b)

FIGURE 1: (a) SEM images of CNTs and (b) experimental setup for fabricating polysiloxane/CNT nanocomposite films.

composites with random filler orientation or using other torque sources, such as shear or a magnetic field, which can only change the orientations of $1 \mathrm{D}$ or $2 \mathrm{D}$ fillers $[16,17,25]$.

Since the first report in 1991 by Iijima [26], CNTs have attracted great interest in many areas of scientific research. There are two forms of CNTs: single-walled CNTs (SWCNTs) and multiwalled CNTs. Tubular structures formed from hexagonal arrangements of carbon atoms [27] give rise to the unique physical properties of CNTs, such as extremely high Young's moduli [28-30], high axial thermal conductivities (about $3000 \mathrm{~W} / \mathrm{mK}$ for multiwalled CNTs even higher for SWCNTs [10, 31], and remarkable electrical properties [18]. These properties make CNTs promising fillers for enhancing the thermal conductivity and other physical properties of polymer-based nanocomposites [32].

However, low-dimensional fillers such as CNTs tend to form agglomerates that have random orientations. This reduces the efficiency of acoustic phonon transfer across polymer-based composite films [33]. Therefore, attaining a homogeneous dispersion of CNTs is important prior to fabricating controlled assemblies of CNT in a polymer [34, 35]. Using CNTs with higher aspect ratios and straightness increases the anisotropy and the thermal conductivity of composites because the fillers form conduction paths at lower filler contents [36]. DC and AC electric fields have been applied when incorporating CNTs in a polymer [10, 18, 37]. In a DC electric field, CNTs move and form a linear dendritic structure that develops and extends from the positive electrode to the negative electrode so that the electrodes eventually become connected by the CNTs in the polymer matris $[24,37]$. When an AC electric field is applied, the CNTs reorient and form network structures oriented perpendicular to the film surface [38]. The modulation of fillers consisting of linear assembles of CNTs (LACNTs) in polymer-based composites has not been studied. Here, we demonstrate that modulating the DC electric field causes LACNTs to form densely packed structures in a polysiloxane matrix, which noticeably enhances the thermal and electrical properties and the optical transmittance of the nanocomposite films.

The four main objectives of this study are to fabricate self-assemblies of CNTs in polymer-based films, to investigate the effects of applying DC and switching DC fields on linear structure formation, to determine the effect of the linear structures on the thermal and electrical properties of the resulting nanocomposites, and to elucidate the formation mechanism of densely packed LACNTs. Homogeneously dispersed CNTs in a prepolymer mixture of polysiloxane were subjected to a DC field or a switching DC field during crosslinking. Scanning electron microscopy (SEM) and digital microscopy observations and thermal diffusivity, UV-Vis transmission, and electrical resistivity measurements were used to characterize the polysiloxane/CNT nanocomposites.

\section{Experimental}

2.1. Materials. Polysiloxane/CNT nanocomposites were prepared by introducing CNTs into a polysiloxane prepolymer mixture. As-purchased CNTs (GSI Creos Co. Ltd., Tokyo, Japan, density: $2.0 \mathrm{~g} / \mathrm{cm}^{3}$ ) were used without further treatment, as shown in Figure 1(a), which confirms that the dimension of CNTs (average length: $5 \mu \mathrm{m}$; inside diameter: 50-70 nm; outer diameter: $80-100 \mathrm{~nm}$ ). Two prepolymers with different viscosities were used to prepare polysiloxane: YE5822(A) (viscosity: 1.2 Pa.s, $\mathrm{Mw}_{\mathrm{av}}$ : 21,000) and YE5822(B) (viscosity: 0.2 Pa.s, $\mathrm{Mw}_{\mathrm{av}}$ : 16,000) (purchased from Momentive Performance Materials). The electrode for applying the electric field was an indium tin oxide (ITO) coated glass slide $\left(2.5 \times 7.5 \times 1.0 \mathrm{~mm}^{3}\right)$ that had a surface resistivity of $8-12 \Omega / \mathrm{sq}$ (Sigma-Aldrich). 
2.2. Fabrication of LACNTs in Polysiloxane/CNT Composites. Polysiloxane/CNT nanocomposites were prepared by the following method (see Figure 1(b)). Various amounts of CNT were incorporated into prepolymer of polysilozane fanging from $0 \sim 0.15 \mathrm{vol} \%$. For example, $0.0011 \mathrm{~g}$ of CNTs $(0.05 \mathrm{vol} \%)$ were distributed in $2 \mathrm{~g}$ of prepolymer YE5822(A) by ultrasonic treatment for $5 \mathrm{~h}$ after mechanical stirring at $1000 \mathrm{rpm}$ for $1 \mathrm{~h} .0 .2 \mathrm{~g}$ of YE5822(B) were introduced into the mixture and sonicated for $5 \mathrm{~min}$ to produce a homogeneous dispersion. This dispersion was placed on a polyamide spacer $\left(1.2 \times 1.2 \mathrm{~mm}^{2} \times 120 \mu \mathrm{m}\right)$, which was placed between two electrodes. DC and switching DC electric fields were applied for $16 \mathrm{~h}$ at room temperature to enhance the orientation and assembly by relocation of the CNTs in the prepolymer suspensions. To switch the DC electric field, the polarity of the electric field was reversed during the $16 \mathrm{~h}$ application of the electric field in four different intervals of $2 \mathrm{~h}$ (seven times), $2 \mathrm{~h} 40 \mathrm{~min}$ (five times), $4 \mathrm{~h}$ (three times), and $8 \mathrm{~h}$ (once). Finally, the prepared composites were dried for $30 \mathrm{~min}$ at $80^{\circ} \mathrm{C}$ to complete curing.

2.3. Characterization and Measurements. The viscosity of prepolymer/CNT suspension was measured using a coneplate (Haake, RheoWin, Thermo Scientific Co.). The surface morphology of the CNT fillers was observed by SEM (JEOL, JSM-6700F). The orientation and linear structure of CNT fillers in the polysiloxane matrix were observed by a digital microscope (Keyence, VHX-9000) after cross-sectional cutting the films. To evaluate the dispersion and orientation, the transmittance of the composites was measured using a UVvisible spectrometer (V630, JASCO). The thermal conductivities of the films were measured using a system for measuring thermal diffusivity based on temperature wave analysis (aiPhase Mobile 1, ai-Phase Co.). The surface resistivity of composite films was measured using a digital multimeter (Advantest R6581) and an AC/DC power supply (Hioki, wt8752) by the two-point probe method, which is capable of measuring high surface resistivities.

\section{Results and Discussion}

Figure 2 shows the viscosity of the prepolymer/CNT suspension as a function of stirring time. The viscosity increases with the degree of cross-linking of the two prepolymers. The viscosity increased very gradually until $2 \mathrm{~h}$ of mixing. It increased rapidly after $2 \mathrm{~h}$ and $30 \mathrm{~min}$ when it increased to 1200 Pa.s over $50 \mathrm{~min}$, the fabrication of a polymer composite film. This is typical behavior during polymerization of a prepolymer suspension. This measurement was performed while mixing the suspension, which was exposed to air. These conditions differ from those used to fabricate nanocomposites in an electric field for which the prepolymer mixture is in a sealed container between two ITO-coated electrodes and the polyimide spacer. Consequently, the viscosity may increase considerably faster than in actual fabrication conditions. However, the trend of the viscosity variation of the prepolymer mixture provides information regarding the basic tendency of the polymer viscosity during cross-linking,

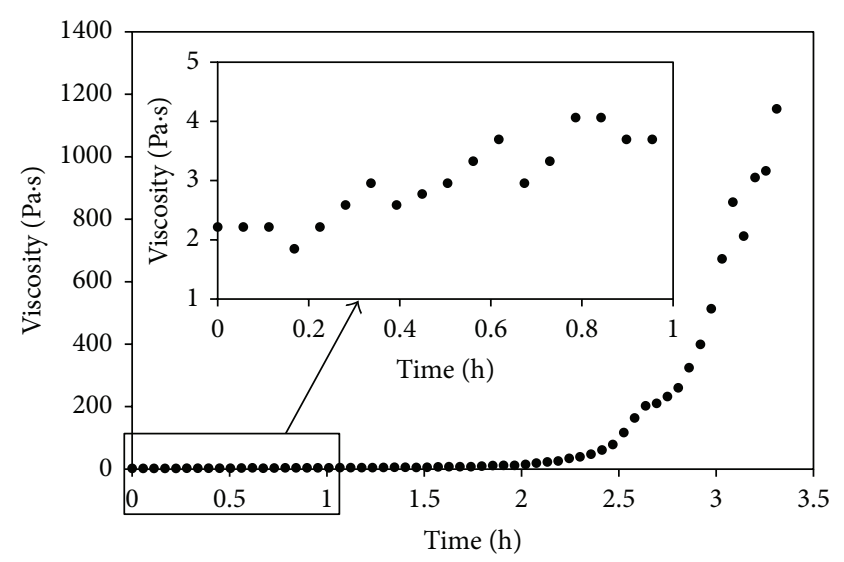

FIGURE 2: Variation of viscosity of prepolymer and CNT suspension as a function of mixing time (inset: magnification of viscosity responses between 0 and $1 \mathrm{~h}$ of mixing time).

which can be used to develop a strategy for controlling the electric field induced torque to improve the orientation and assembly of CNTs in the polymer matrix. The observed tendency implies that CNT assembly finishes before the steep increase in the viscosity. Complete cross-linking of the polysiloxane prepolymer was indirectly determined from the DC breakdown voltage [39]. The breakdown voltage increases as a function of the polymerization of the prepolymer suspension; it stabilized after applying an electric field for $16 \mathrm{~h}$, which corresponds to the completion of polymerization.

Figure 3 shows surface and cross-sectional micrographs of polysiloxane/CNT nanocomposites fabricated under different electric fields. The dark microscale domains of CNTs indicated by the white circles in the surface micrographs in Figures 3(a1)-3(c1) reveal that many CNTs agglomerate. These agglomerates are homogeneously distributed over a smaller scale in the polymer without the application of an electric field. They form linearly aligned assembly when an electric field is applied Figures 3(b2)-3(c2). Applying DC (Figure 3(b)) and switching DC (Figure 3(c)) electric fields gives quite different CNT structures in the polymer matrix, as both the surface and cross-sectional images show. After applying DC and switching DC electric fields, the dark domains, which correspond to CNTs agglomerates and tips of LACNTs that anchor the film surfaces, were observed on the surfaces of the nanocomposite films. While applying both DC and switching DC fields causes the fabrication of LACNTs, the LACNT population and the CNT density in the linear structures vary depending on the applied electric field.

Comparison of the cross-sectional images in Figures 3(b2) and 3(c2) reveals that the LACNTs fabricated under a switching DC field are thicker and darker than those fabricated under a DC field. The slightly lower number of identifiable LACNTs produced by the DC field compared with the switching DC field indicates that some LACNTs were incorporated in other LACNTs, increasing their thickness. This gives rise to the denser packing of CNTs in LACNTs. The interconnected LACNTs networks indicated by the circles in Figure 3(c) may be related to traces 


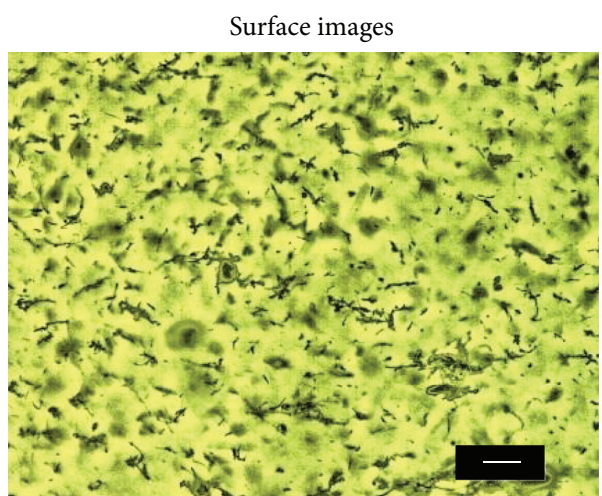

(a1)

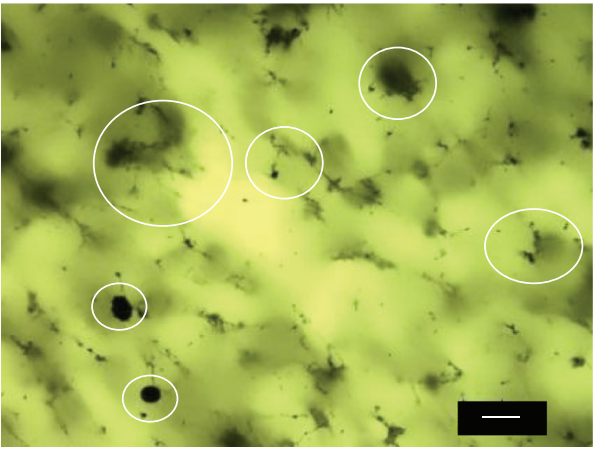

(b1)

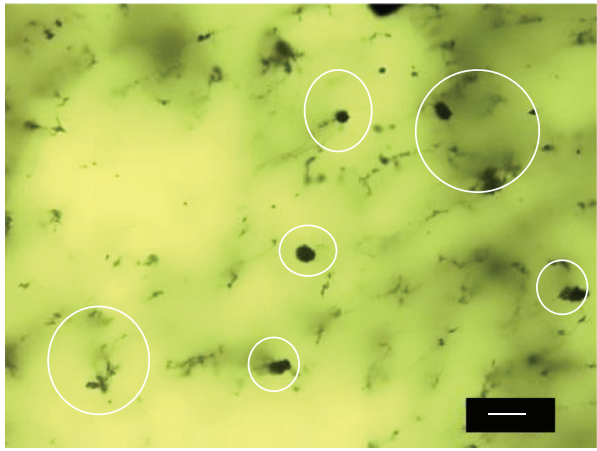

(c1)

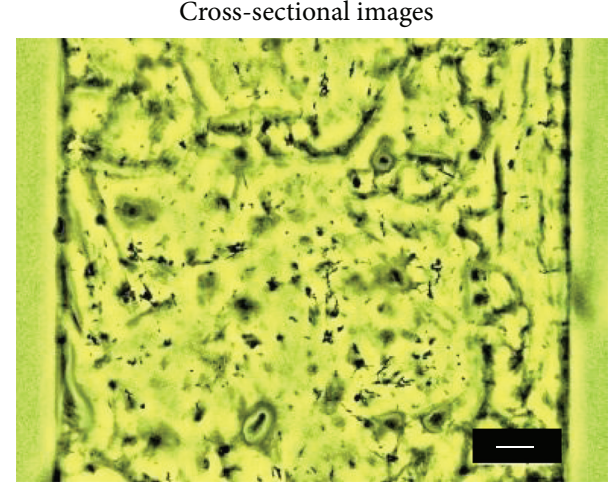

(a2)

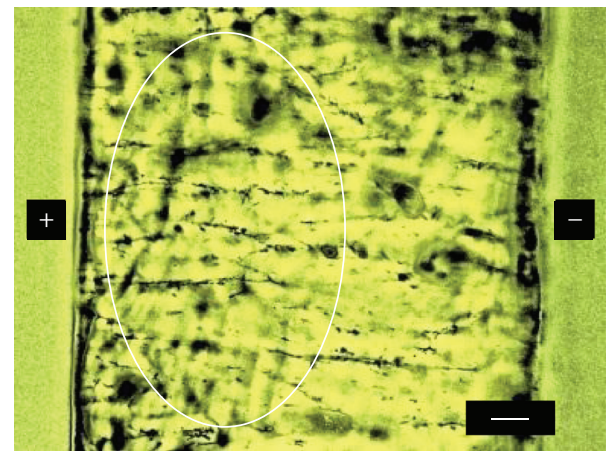

(b2)

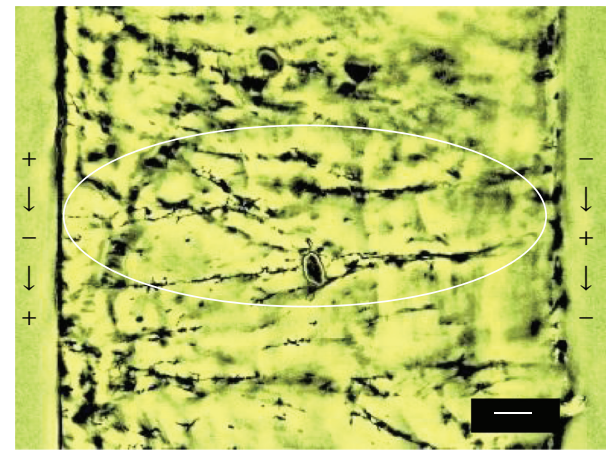

(c2)

FIGURE 3: Digital micrographs of polysiloxane/CNT nanocomposites prepared by applying different electric fields (CNT: 0.05 vol\%; scale bar: $20 \mu \mathrm{m}$ ); (a) no electric field, (b) DC electric field, (c) SWDC_4 h (three times).

formed during incorporation of LACNTs when switching the electric field. Comparison of the surface images for DC and switching DC shows that domains with fewer but thicker CNT agglomerates are obtained by the switching DC field. This implies that applying a switching DC field increases the CNT population in LACNTs. When an electric field is applied, polarized 1D or 2D dielectric fillers tend to orient their longitudinal directions parallel to the electric flux to minimize the electrostatic energy [16]. The polarized ends of CNTs become interconnected through Coulomb attraction forming a side-by-side attachment of CNTs due to self-assembly and generating a dendritic structure of CNTs [37]. This structure in which one end is localized to the anode electrode is typical when a DC electric field is applied [24]. An increased thickness in LACNT bundles has previously been observed when CNT surfaces were modified with tetraoctylammonium and bromide ions for charge stabilization in an electric field that was alternately switched on and off [20]. However, there have been no reports of controlling LACNT thickness by just modulating external torque sources. Thus, the formation of LACNTs that anchor the composite surfaces and the increase in the LACNT thickness on applying a switching DC field are a novel observation.

To investigate the modulation of LACNT structures in a switching DC electric field, the polysiloxane/CNT suspensions were subjected to DC fields with different switching intervals, as shown in Figure 4. For a DC switching with 


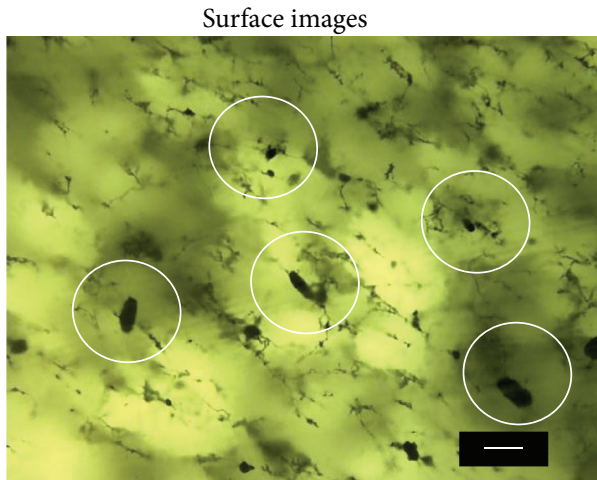

(a1)

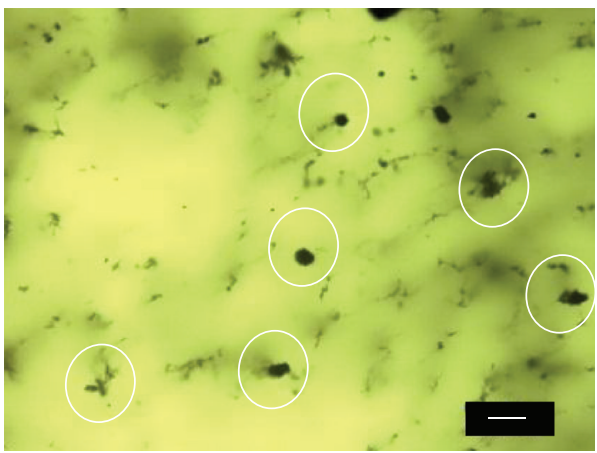

(b1)

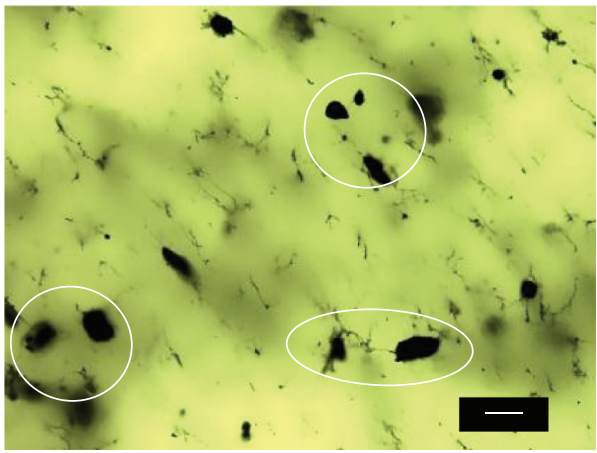

$(\mathrm{c} 1)$

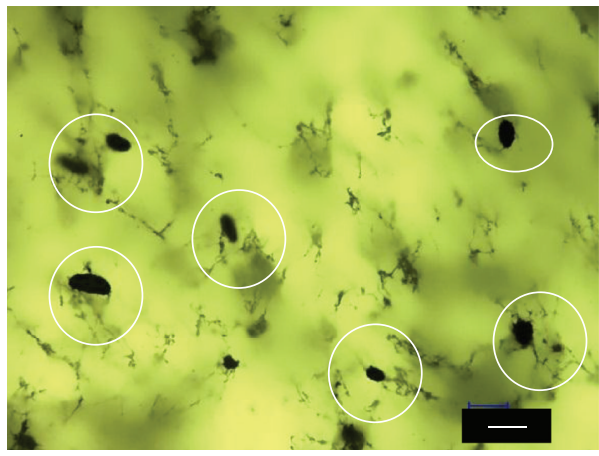

(d1)

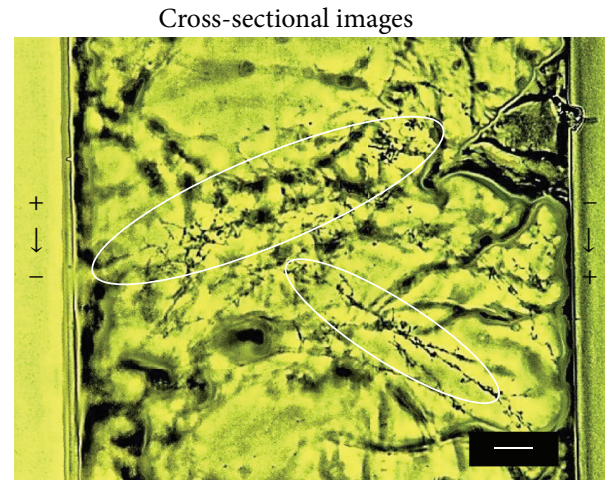

(a2)

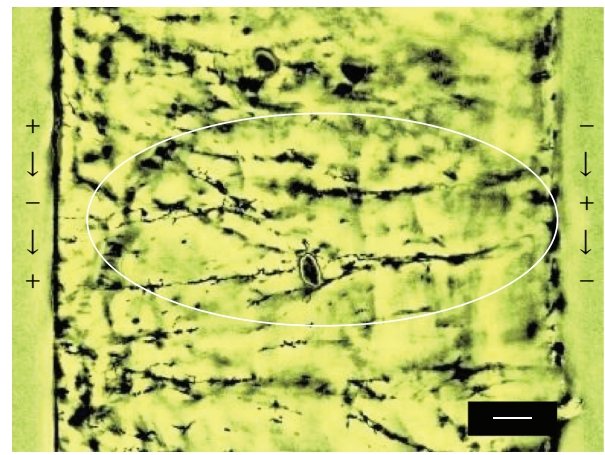

(b2)

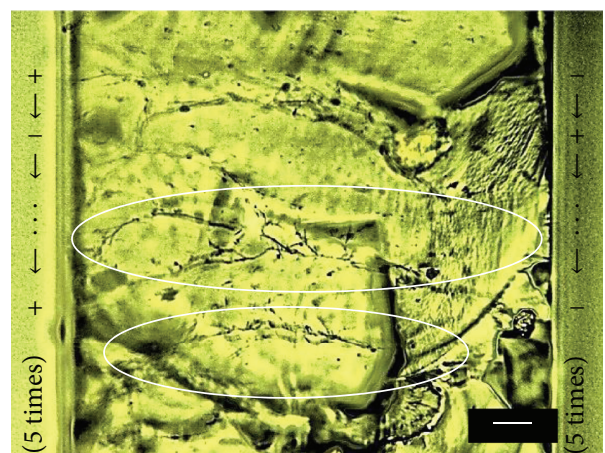

(c2)

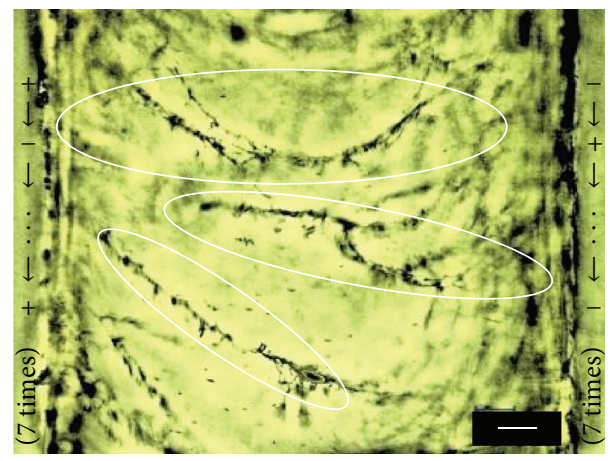

(d2)

FIGURE 4: Digital micrographs of polysiloxane/CNT nanocomposites prepared for DC fields with different switching intervals (CNT: 0.05 vol\%; scale bar: $20 \mu \mathrm{m}$ ); (a) SWDC50V_8 h, (b) SWDC50V_4 h, (c) SWDC50V_2 h 40 min, and (d) SWDC50V_2 h. 


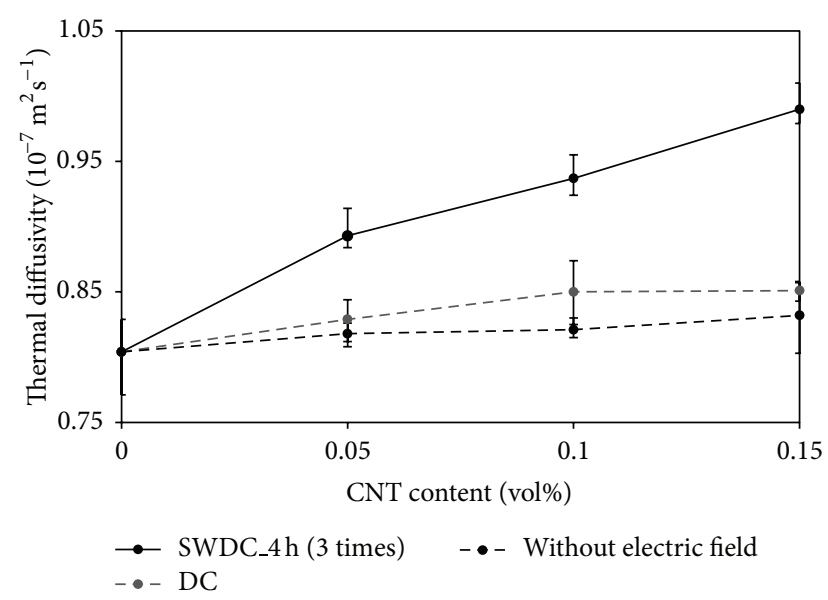

(a)

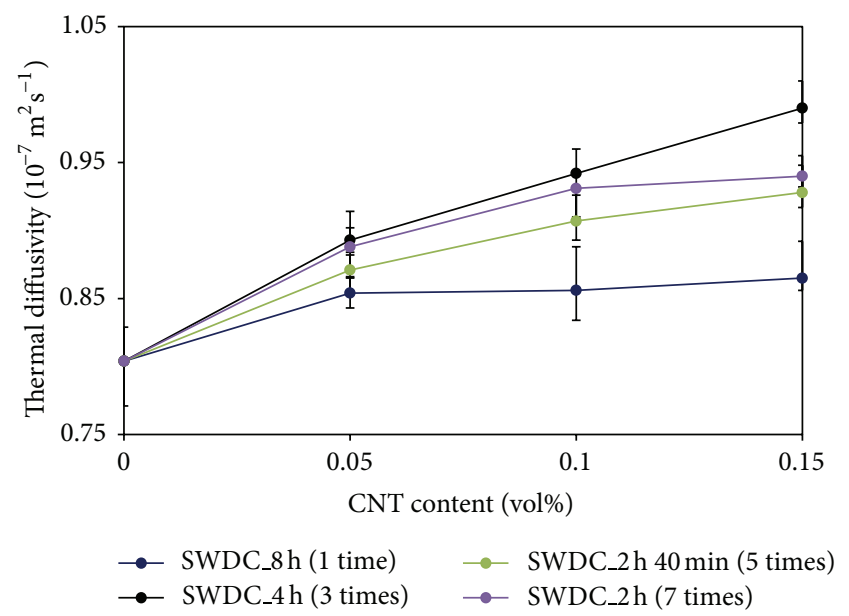

(b)

FIGURE 5: Thermal diffusivities of nanocomposites as a function of CNT content under (a) different electric fields and (b) switching DC times.

an $8 \mathrm{~h}$ interval, the LACNT tips are located on the righthand side surface of the film where the positive electrode was located (Figure 4(a1)). With increasing switching interval, the thickness of ramified LACNTs that anchor the film surfaces is increased (Figures 4(b2)-4(d2)). The cross-sectional images indicate that the reduction in the number of LACNTs is directly related to the increase in the LACNT thickness. This implies that LACNTs with low CNT densities were incorporated in neighboring LACNTs, resulting in the formation of LACNTs with a higher CNT density. The darker and thicker domains of CNT agglomerates in the surface images also indicate that the CNT concentration of LACNTs increases with increasing DC switching interval. The CNT density of agglomerates was the highest for switching DC (SWDC) of $8 \mathrm{~h}$ (Figure 4(b1)). However, the CNT concentration started to decrease with increasing switching time, resulting in denser CNT agglomerates.

The thermal diffusivity was measured to investigate the cause of the variation in LACNT structures fabricated under different electric fields (Figure 5). The thermal diffusivity

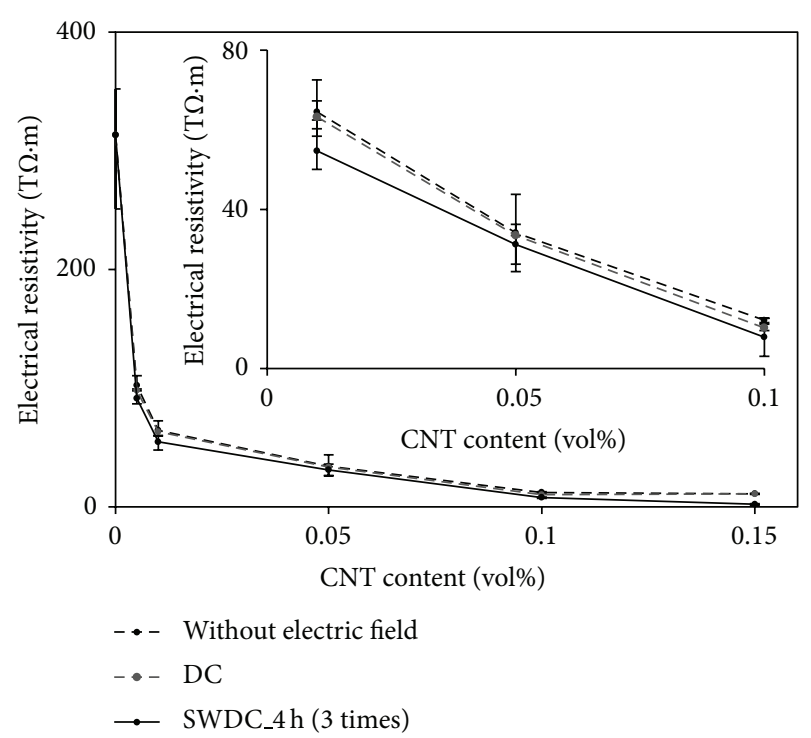

FIGURE 6: Electrical resistivities of nanocomposites as a function of CNT content under different electric fields (inset: magnification of resistivity responses between 0 and 0.1 vol\% of CNT content).

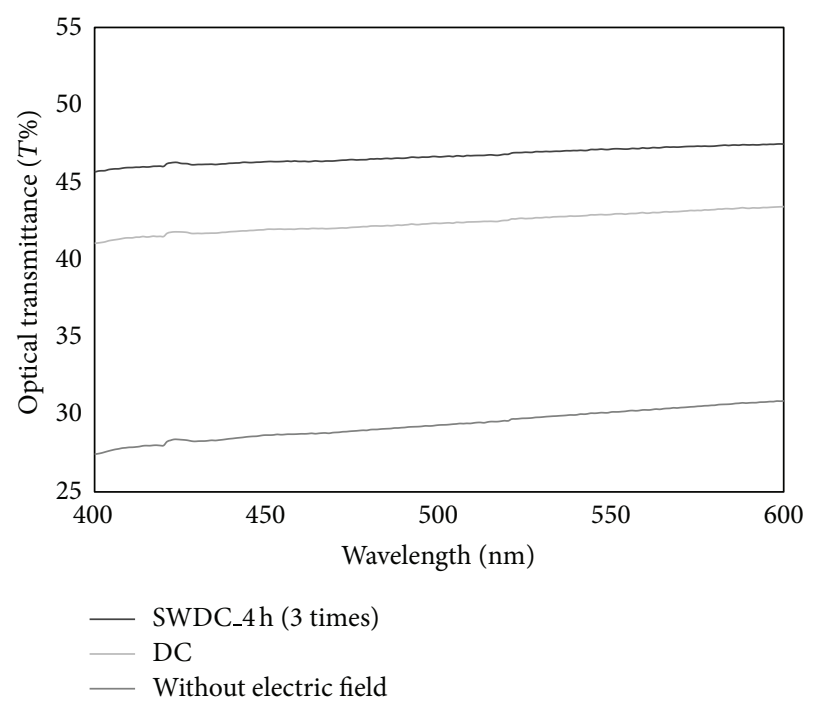

FIGURE 7: UV-Vis transmittance of nanocomposites under different electric fields (CNT: 0.05 vol\%).

of each nanocomposite film was measured five times and the average value was obtained. Two different electric field structures were applied: one involving the application of a DC field or a switching DC field (Figure 5(a)) and the other involving varying the switching interval during application of a switching DC field (Figure 5(b)). The thermal diffusivity increased proportionally with increasing CNT content for both DC and switching DC fields (Figure 5(a)). The diffusivity was enhanced by a factor of almost five times when a switching DC field and no electric field were applied compared to when a DC field and no electric field were applied. This enhancement in the thermal diffusivity on the application of a switching DC field is related to the variation 

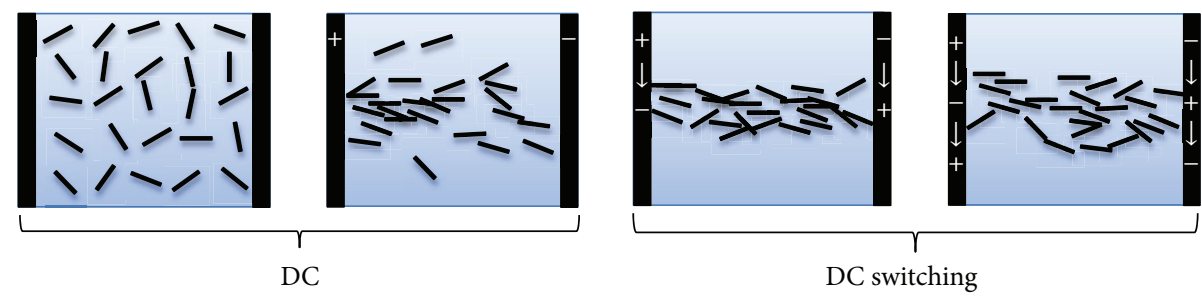

(a)

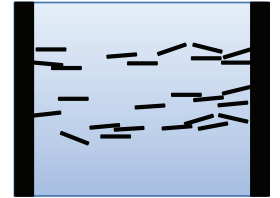

SWDC_8h (1 time)

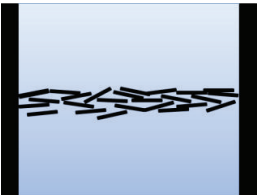

SWDC_4h (3 times)

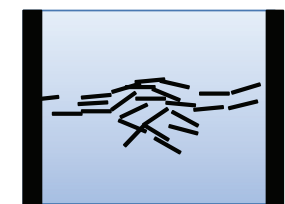

SWDC_2 $\mathrm{h} 40 \mathrm{mins}$ (5 times)

(b)
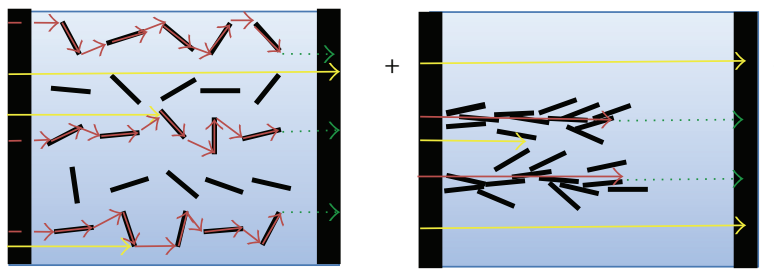

DC

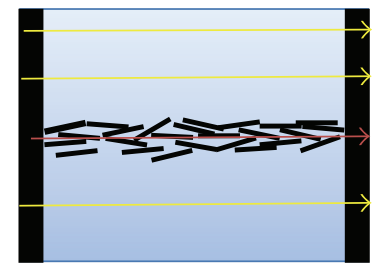

SWDC_4h (3 times)

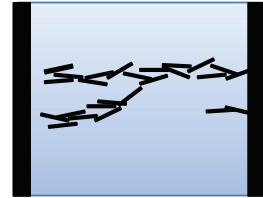

SWDC_2 $\mathrm{h}$ (7 times) 
This means that the fabrication of densely packed linear structures with conducting CNTs simultaneously enhances the thermal diffusivity and the electrical conductivity.

The LACNTs also affect the optical properties of the composites, as indicated by the variation in the optical transmittance shown in Figure 7. A homogeneous dispersion of CNTs in the polymer matrix obtained when no electric field was applied gave the lowest transmittance. The composite films oriented by applying DC and switching DC electric fields had higher transmittances. Visible light introduced from one surface of the film will either be absorbed or scattered in a different direction when it encounters a surface of an inorganic nanofiller in the polymer matrix. The formation of a linear dendritic or network structure by CNT movement can be induced by the combination of Columbic attraction and electrophoresis in a polymer [20]. The CNTs that migrate leave spaces in the polymer matrix, which permit UV and visible light to transmit though the film planes.

Figure 8 illustrates the mechanisms for (a) fabricating LACNTs with densely packed CNTs under a switching DC field, (b) optimizing LACNTs by varying the DC switching interval, and (c) enhancing the thermal, electrical, and optical properties using LACNTs. When a DC electric field is applied, the longitudinal ends of the conducting $1 \mathrm{D}$ nanofillers are depolarized [16] and fillers with high aspect ratios orient their longitudinal ends parallel to the direction of the electric flux to realize electrical stabilization [24] (see Figure 8(a)). For a low-viscosity prepolymer such as polysiloxane, the oriented CNTs start to move toward the positive electrode by electrophoresis. At the same time, the depolarized ends of the CNTs will be attracted to each other by Coulombic attraction resulting in end-to-end attachment [24], which will facilitate the formation of linear dendritic or network structures of CNTs on application of an electric field. By repeatedly switching the DC field, the LACNTs with low CNT densities will be attracted to each other. CNTs that do not form LACNTs during previous application of a DC electric field will join to form denser LACNTs due to Columbic attraction. Repeated switching will result in the formation of LACNT-to-LACNT interconnections, changing the linear structure from a dendritic structure to a network structure, as the cross-sectional images in Figures 4(b)-4(d) show. These interconnections between groups of LACNTs may give rise to a narrower filler-to-filler attachment in the polymer, if the prepolymer viscosity is kept constant. However, the prepolymer viscosity typically increases abruptly over a short time as polymerization proceeds, as shown in Figure 2. (Note that the rate of increase of the viscosity in Figure 2 is over four times higher than that of an actual experimental setup, which is performed in a sealed container with no exposure to air when the electric field is applied. The test data is presented to show the tendency of the polymer viscosity to vary with cross-linking.) Consequently, excessive switching of the DC field will reduce the number of LACNTs (see Figure 8(b)), especially when DC field switching occurs as the viscosity of the polymer matrix is increasing. This implies that it is necessary to optimize the DC switching interval relative to the variation of the viscosity of the prepolymer matrix. Modifying the CNT surface can cause the linear structure to develop into a denser bundle structure [37], as has been demonstrated by cycling the electric field on and off in tetrahydrofuran [20]. Orientating conducting fillers parallel to the heat flux enhances the thermal conduction relative to a random distribution [10] because it increases the number of heat conduction paths through the conducting filler. Such enhancement of the thermal and electrical conductivities and the optical transmittance can be realized by fabricating linear LACNTs and by developing structures that anchor film surfaces by realizing dense packing, as illustrated in Figure 8(c). Fabricating densely packed LACNTs by switching a DC field can increase the thermal and electrical conductivities while using less filler than conventional methods.

\section{Conclusion}

Densely packed LACNTs were fabricated in a polymer-based nanocomposite film by applying and modulating DC electric fields. Linear-assembly of CNTs was demonstrated through Coulombic attraction and electrophoresis by controlling the viscosity of the prepolymer mixture. The application of a switching DC field caused the linear structures to form densely packed structures. On the application of a DC field, the CNT fillers formed a dendritic structure, which developed into densely packed LACNTs when the direction of the DC electric field was switched at a controlled switching interval. The resulting LACNTs enhanced the thermal and electrical conductivities and the optical transmittance of the polysiloxane/CNT nanocomposites. It is thus a promising technique for fabricating polymer-based materials with high thermal and electrical conductivities without using surface modification or increasing the conducting filler content.

\section{Acknowledgments}

The authors are grateful for support from the New Energy and Industrial Technology Development Organization (NEDO) of Japan, the Ultra Composite Material Technology Development Project ("Ultra Composite"), and the Global Research Lab. (GRL) Program of the National Research Foundation of Korea (NRF) funded by the Ministry of Education, Science and Technology (MEST) of Korea (Grant no. 2010-00339).

\section{References}

[1] V. Choudhary and A. Gupta, "Polymer/carbon nanotube nanocomposites," in Carbon Nanotubes-Polymer Nanocomposites, S. Yellampalli, Ed., Intech, 2011.

[2] W. S. Lee and J. Yu, "Comparative study of thermally conductive fillers in underfill for the electronic components," Diamond and Related Materials, vol. 14, no. 10, pp. 1647-1653, 2005.

[3] J. Deng, X. Ding, W. Zhang et al., "Carbon nanotube-polyaniline hybrid materials," European Polymer Journal, vol. 38, no. 12, pp. 2497-2501, 2002.

[4] B. S. Shim, J. Starkovich, and N. Kotov, "Multilayer composites from vapor-grown carbon nano-fibers," Composites Science and Technology, vol. 66, no. 9, pp. 1171-1178, 2006.

[5] Z. M. Huang, Y. Z. Zhang, M. Kotaki, and S. Ramakrishna, "A review on polymer nanofibers by electrospinning and their 
applications in nanocomposites," Composites Science and Technology, vol. 63, no. 15, pp. 2223-2253, 2003.

[6] H. B. Cho, T. Nakayama, T. Suzuki et al., "Linear assembles of $\mathrm{BN}$ nanosheets, fabricated in polymer/BN nanosheet composite film," Journal of Nanomaterials, vol. 2011, Article ID 693454, 7 pages, 2011.

[7] S. Kemaloglu, G. Ozkoc, and A. Aytac, "Properties of thermally conductive micro and nano size boron nitride reinforced silicon rubber composites," Thermochimica Acta, vol. 499, no. 1-2, pp. 40-47, 2010.

[8] X. L. Xie, Y. W. Mai, and X. P. Zhou, "Dispersion and alignment of carbon nanotubes in polymer matrix: a review," Materials Science and Engineering R, vol. 49, no. 4, pp. 89-112, 2005.

[9] Y. S. Song and J. R. Youn, "Influence of dispersion states of carbon nanotubes on physical properties of epoxy nanocomposites," Carbon, vol. 43, no. 7, pp. 1378-1385, 2005.

[10] Z. Han and A. Fina, "Thermal conductivity of carbon nanotubes and their polymer nanocomposites: a review," Journal of Nanomaterials, vol. 36, no. 7, pp. 914-944, 2011.

[11] W. Zhao, H. Wang, H. Tang, and G. Chen, "Facile preparation of epoxy-based composite with oriented graphite nanosheets," Polymer, vol. 47, no. 26, pp. 8401-8405, 2006.

[12] W. Zhou, S. Qi, H. Li, and S. Shao, "Study on insulating thermal conductive BN/HDPE composites," Thermochimica Acta, vol. 452, no. 1, pp. 36-42, 2007.

[13] E. Logakis, C. H. Pandis, P. Pissis, J. Pionteck, and P. Pötschke, "Highly conducting poly(methyl methacrylate)/carbon nanotubes composites: investigation on their thermal, dynamicmechanical, electrical and dielectric properties," Composites Science and Technology, vol. 71, no. 6, pp. 854-862, 2011.

[14] H. B. Cho, T. Nakayama, T. Suzuki et al., "Formation and structural characteristic of perpendicularly aligned boron nitride nanosheet bridges in polymer/boron nitride composite film and its thermal conductivity," Japanese Journal of Applied Physics, vol. 50, no. 1, Article ID 01BJ05, 2011.

[15] M. H. Al-Saleh and U. Sundararaj, "A review of vapor grown carbon nanofiber/polymer conductive composites," Carbon, vol. 47, no. 1, pp. 2-22, 2009.

[16] J. Lu, W. Weng, X. Chen, D. Wu, C. Wu, and G. Chen, "Piezoresistive materials from directed shear-induced assembly of graphite nanosheets in polyethylene," Advanced Functional Materials, vol. 15, no. 8, pp. 1358-1363, 2005.

[17] N. Toyohara, Y. Benino, T. Fujiwara et al., "Enhancement and depression in second-order optical nonlinearity of $\mathrm{Ba}_{2} \mathrm{TiGe}_{2} \mathrm{O}_{8}$ in crystallized glass prepared in a high magnetic field," Journal of Applied Physics, vol. 99, no. 4, 2006.

[18] X. Q. Chen, T. Saito, H. Yamada, and K. Matsushige, "Aligning single-wall carbon nanotubes with an alternating-current electric field," Applied Physics Letters, vol. 78, no. 23, pp. 3714-3716, 2001.

[19] T. Takahashi, T. Murayama, A. Higuchi, H. Awano, and K. Yonetake, "Aligning vapor-grown carbon fibers in polydimethylsiloxane using dc electric or magnetic field," Carbon, vol. 44, no. 7, pp. 1180-1188, 2006.

[20] P. V. Kamat, K. G. Thomas, S. Barazzouk, G. Girishkumar, K. Vinodgopal, and D. Meisel, "Self-assembled linear bundles of single wall carbon nanotubes and their alignment and deposition as a film in a dc field," Journal of the American Chemical Society, vol. 126, no. 34, pp. 10757-10762, 2004.

[21] T. Saotome, H. Kim, D. Lashmore et al., "Transparent conducting film: effect of mechanical stretching to optical and electrical properties of carbon nanotube mat," Bulletin of Materials Science, vol. 34, no. 4, pp. 615-622, 2011.

[22] K. Mylvaganam and L. C. Zhang, "Fabrication and application of polymer composites comprising carbon nanotubes," Recent Patents on Nanotechnology, vol. 1, no. 1, pp. 59-65, 2007.

[23] Y. Zhang, A. Chang, J. Cao et al., "Electric-field-directed growth of aligned single-walled carbon nanotubes," Applied Physics Letters, vol. 79, no. 19, pp. 3155-3157, 2001.

[24] C. A. Martin, J. K. W. Sandler, A. H. Windle et al., "Electric fieldinduced aligned multi-wall carbon nanotube networks in epoxy composites," Polymer, vol. 46, no. 3, pp. 877-886, 2005.

[25] L. Chen, G. Chen, and L. Lu, "Piezoresistive behavior study on finger-sensing silicone rubber/graphite nanosheet nanocomposites," Advanced Functional Materials, vol. 17, no. 6, pp. 898904, 2007.

[26] S. Iijima, "Helical microtubules of graphitic carbon," Nature, vol. 354, no. 6348, pp. 56-58, 1991.

[27] R. Khare and S. Bose, "Carbon nanotube based compositesa review," Journal of Minerals \& Materials Characterization \& Engineering, vol. 4, pp. 31-46, 2005.

[28] A. L. Kalamkarov, A. V. Georgiades, S. K. Rokkam, V. P. Veedu, and M. N. Ghasemi-Nejhad, "Analytical and numerical techniques to predict carbon nanotubes properties," International Journal of Solids and Structures, vol. 43, no. 22-23, pp. 68326854, 2006.

[29] J. P. Salvetat-Delmotte and A. Rubio, "Mechanical properties of carbon nanotubes: a fiber digest for beginners," Carbon, vol. 40, no. 10, pp. 1729-1734, 2002.

[30] R. S. Ruoff, D. Qian, and W. K. Liu, "Mechanical properties of carbon nanotubes: theoretical predictions and experimental measurements," Comptes Rendus Physique, vol. 4, no. 9, pp. 9931008, 2003.

[31] F. Deng, Q. S. Zheng, L. F. Wang, and C. W. Nan, "Effects of anisotropy, aspect ratio, and nonstraightness of carbon nanotubes on thermal conductivity of carbon nanotube composites," Applied Physics Letters, vol. 90, no. 2, Article ID 021914, 2007.

[32] M. J. Biercuk, M. C. Llaguno, M. Radosavljevic, J. K. Hyun, A. T. Johnson, and J. E. Fischer, "Carbon nanotube composites for thermal management," Applied Physics Letters, vol. 80, no. 15, pp. 2767-2769, 2002.

[33] J. I. Paredes and M. Burghard, "Dispersions of individual singlewalled carbon nanotubes of high length," Langmuir, vol. 20, no. 12, pp. 5149-5152, 2004.

[34] Z. Ounaies, C. Park, K. E. Wise, E. J. Siochi, and J. S. Harrison, "Electrical properties of single wall carbon nanotube reinforced polyimide composites," Composites Science and Technology, vol. 63, no. 11, pp. 1637-1646, 2003.

[35] J. Sandler, M. S. P. Shaffer, T. Prasse, W. Bauhofer, K. Schulte, and A. H. Windle, "Development of a dispersion process for carbon nanotubes in an epoxy matrix and the resulting electrical properties," Polymer, vol. 40, no. 21, pp. 5967-5971, 1999.

[36] O. Breuer and U. Sundarara, "Big returns from small filber: a review of polymer/carbon nanotube composites," Polymer Composties, vol. 25, pp. 630-641, 2004.

[37] M. Senthil Kumar, T. H. Kim, S. H. Lee et al., "Influence of electric field type on the assembly of single walled carbon nanotubes," Chemical Physics Letters, vol. 383, no. 3-4, pp. 235239, 2004.

[38] T. Prasse, J. Y. Cavaillé, and W. Bauhofer, "Electric anisotropy of carbon nanofibre/epoxy resin composites due to electric field 
induced alignment," Composites Science and Technology, vol. 63, no. 13, pp. 1835-1841, 2003.

[39] H. B. Cho, T. Nakayama, Y. Tokoi et al., "Facile preparation of a polysiloxane-based hybrid composite with highly-oriented boron nitride nanosheets and an unmodified surface," Composites Science and Technology, vol. 70, no. 12, pp. 1681-1686, 2010.

[40] C. Zhang, P. Wang, C. A. Ma, G. Wu, and M. Sumita, “Temperature and time dependence of conductive network formation: dynamic percolation and percolation time," Polymer, vol. 47, no. 1, pp. 466-473, 2006.

[41] H. Tantang, J. Y. Ong, C. L. Loh et al., "Using oxidation to increase the electrical conductivity of carbon nanotube electrodes," Carbon, vol. 47, no. 7, pp. 1867-1870, 2009. 

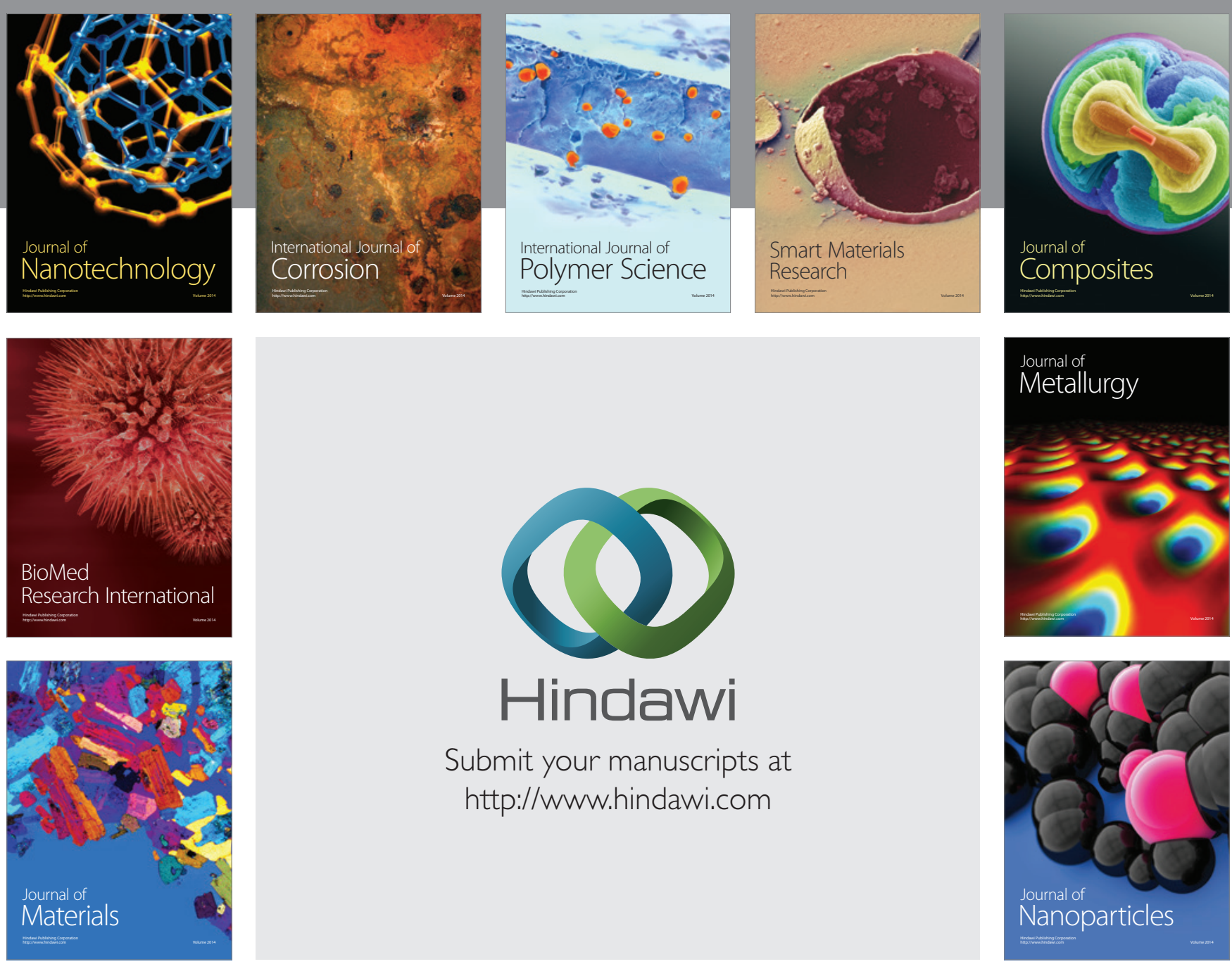

Submit your manuscripts at http://www.hindawi.com
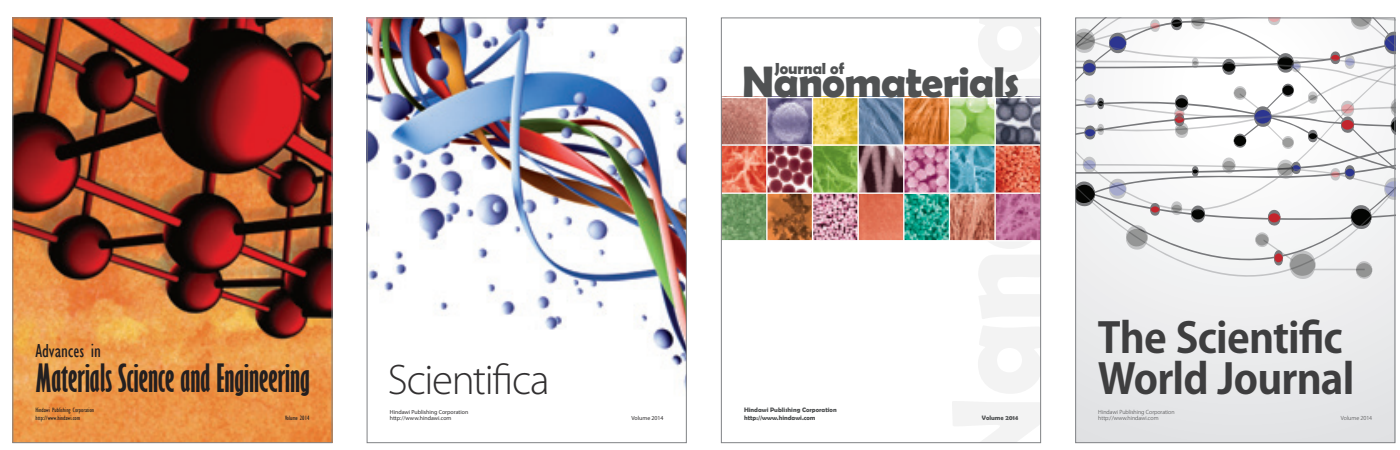

\section{The Scientific World Journal}
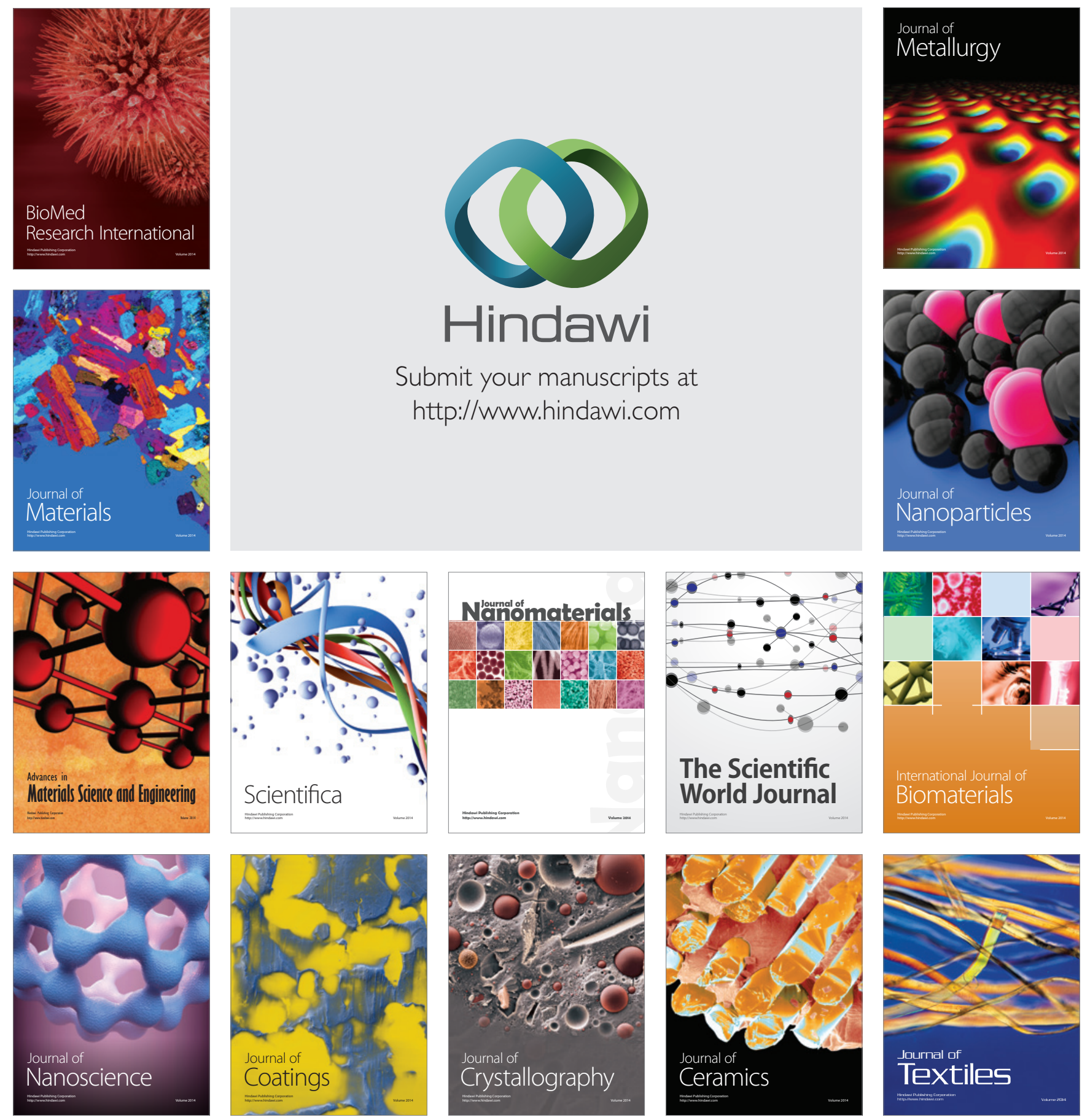